\title{
Motor and Dorsal Root Ganglion Axons Serve as Choice Points for the Ipsilateral Turning of dI3 Axons
}

\author{
Oshri Avraham, ${ }^{1}$ Yoav Hadas, ${ }^{1}$ Lilach Vald, ${ }^{1}$ Seulgi Hong, ${ }^{2}$ Mi-Ryoung Song, ${ }^{2}$ and Avihu Klar ${ }^{1}$ \\ ${ }^{1}$ Department of Medical Neurobiology, Institute for Medical Research Israel-Canada, Hebrew University-Hadassah Medical School, Jerusalem, Israel \\ 91120, and 2BioImaging Research Center and Cell Dynamics Research Center, Gwangju Institute of Science and Technology, Gwangju 500-712, \\ Republic of Korea
}

The axons of the spinal intersegmental interneurons are projected longitudinally along various funiculi arrayed along the dorsal-ventral axis of the spinal cord. The roof plate and the floor plate have a profound role in patterning their initial axonal trajectory. However, other positional cues may guide the final architecture of interneuron tracks in the spinal cord. To gain more insight into the organization of specific axonal tracks in the spinal cord, we focused on the trajectory pattern of a genetically defined neuronal population, dI 3 neurons, in the chick spinal cord. Exploitation of newly characterized enhancer elements allowed specific labeling of dI3 neurons and axons. dI3 axons are projected ipsilaterally along two longitudinal fascicules at the ventral lateral funiculus (VLF) and the dorsal funiculus (DF). dI3 axons change their trajectory plane from the transverse to the longitudinal axis at two novel checkpoints. The axons that elongate at the DF turn at the dorsal root entry zone, along the axons of the dorsal root ganglion (DRG) neurons, and the axons that elongate at the VLF turn along the axons of motor neurons. Loss and gain of function of the Lim-HD protein Isl1 demonstrate that Isl1 is not required for dI3 cell fate. However, Isl1 is sufficient to impose ipsilateral turning along the motor axons when expressed ectopically in the commissural dI1 neurons. The axonal patterning of dI3 neurons, revealed in this study, highlights the role of established axonal cues-the DRG and motor axons-as intermediate guidepost cues for dI3 axons.

\section{Introduction}

Spinal sensory neurons derive from several populations of interneurons (INs) in the embryonic dorsal spinal cord that are distinguished by a transcriptional code, the positions of somata, and their axonal patterning (Lee and Jessell, 1999; Helms and Johnson, 2003). In the adult spinal cord, the intersegmental INs project their axons longitudinally in the white matter along defined funiculi arrayed along the dorsal-ventral axis. Anatomical and physiological studies have attributed different sensory modalities to specific longitudinally projecting funiculi in the white matter. Thus, it is likely that, in the white matter, the longitudinally projecting axons are arranged in an IN-specific manner. For example, the axons of dI1 and dI2 neurons are arranged in a tight IN-specific bundle at distinct dorsoventral positions at the lateral funiculus (LF), where the dI2 fascicule is located dorsally to the

Received May 10, 2010; revised Aug. 20, 2010; accepted Sept. 20, 2010.

This work was supported by grants to A.K. from the Israel Science Foundation (229/09), The Legacy Heritage Biomedical Science Partnership Program of the Israel Science Foundation (Grant 1930/08), DFG (German Research Foundation), and the Israel Ministry of Health, and by grants to M.S. from the Biolmaging Research Center at Gwangju Institute of Science and Technology and the National Research Foundation of Korea funded by the Ministry of Education, Science, and Technology (E00343). We thank Sylvia Evans for the IsI 1 hypo mice, Thomas Jessell for Isl1, the Lhx1/5 monoclonal antibody, and Lhx2/9 and Lhx1/5 rabbit antibodies, Carmen Birchmeier for the tlx3 antibody, Chaya Klacheim for the BEN antibody, Artur Kania for the chick Is/1 gene, Axel Visel for help with initia characterization of enhancers in mice, and Adi Schejter and Oksana Cohen for technical assistance. We are also grateful to Samantha Butler, Oded Behar, and Alexander Binshtok for comments on the manuscript.

Correspondence should be addressed to Avihu Klar, Department of Medical Neurobiology, Institute for Medical Research Israel-Canada, Hebrew University-Hadassah Medical School, P.0. Box 12272, Jerusalem, Israel 91120. E-mail: avihu@cc.huji.ac.il.

DOI:10.1523/JNEUROSCI.2380-10.2010

Copyright $\odot 2010$ the authors $\quad 0270-6474 / 10 / 3015546-12 \$ 15.00 / 0$
dI1 fascicule (Avraham et al., 2009). IN may fasciculate together homophilically by expressing a unique code of adhesion molecules. Support for a possible homophilic interaction between axons of the same subtype arises from the observation that $\mathrm{dI} 1_{\text {comm }}$ axons, which are projected contralaterally across the midline, and $\mathrm{dI} 1_{\mathrm{ipsi}}$ axons, which elongate at the ipsilateral side, fasciculate together as they extend rostrally at the LF (Avraham et al., 2009). As for positional cues, a role for B-class ephrins in specifying the dorsoventral position of longitudinally projecting commissural axons has been suggested. B-class ephrins are expressed in the dorsal-half neural tubes. Perturbation of EphB signaling causes a dorsal overshooting of the dorsal commissural INs, suggesting that the dorsal boundary of the LF is limited by B-class ephrins (Imondi and Kaprielian, 2001). However, little is known about cellular and molecular cues that instruct the position and partitioning of each IN population.

In the current study, we describe the axonal pathways of $\mathrm{dI} 3$ neurons in the chick neural tube. Specific labeling of dI 3 neurons was attained using enhancer intersections between newly characterized enhancer elements. dI3 neurons project their axons ipsilaterally and longitudinally in two fascicules: a ventral fascicule at the ventral lateral funiculus (VLF) and a dorsal fascicule at the dorsal funiculus (DF). Accordingly, dI3 neurons are subdivided to dorsally and ventrally projecting neurons. Two novel choice points at intermediate targets, at which $\mathrm{dI} 3$ neurons change the plane of their axonal trajectory, are illustrated. The dorsally projecting dI3 axons change their trajectory from the transverse to the longitudinal plane as they encounter axons of the dorsal root ganglion (DRG) neurons at the dorsal root entry zone (DREZ). 
The ventrally projecting dI3 axons change their trajectory plane from the ventral to the lateral plane as they encounter the axons of the motor neurons. To gain insight into the possible molecular mechanisms controlling the unique dI3 axonal patterning, we focused on the dI3-specific Lim-HD transcription factor Isl1. Isl1 is not required for $\mathrm{dI} 3$ cell fate, as demonstrated by loss- and gain-of-function experiments. However, Isl1 is sufficient to instruct ipsilateral axonal projection along the motor axons to the contralaterally projecting $\mathrm{dI} 1_{\text {comm }}$ neurons when expressed ectopically in dI1 neurons.

\section{Materials and Methods}

In ovo electroporations. Fertilized White Leghorn chicken eggs were incubated at $38.5-39^{\circ} \mathrm{C}$. A DNA solution of $5 \mathrm{mg} / \mathrm{ml}$ was injected into the lumen of the neural tube at either Hamburger-Hamilton $(\mathrm{HH})$ stages 12-14 (CMV enhancer in pCAGG plasmid) or stages $17-18(215,242$, and 586 enhancers) Electroporation was performed using $3 \times 50 \mathrm{~ms}$ pulses at $25 \mathrm{~V}$, applied across the embryo using a $0.5 \mu \mathrm{m}$ tungsten wire and a BTX electroporator (ECM 830). Embryos were incubated for 2-3 d before analysis (Avraham et al., 2010).

Spinal cord open-book preparation. Embryonic day 6 (E6) electroporated chick spinal cord tissues were prepared as an open-book preparation by making a longitudinal incision along the roof plate with a sharp tungsten microneedle from the hindbrain down to the tail. The DRGs were then separated from the spinal cord, leaving the floor plate intact. The hindlimb and forelimb were marked by charcoal powder, and then the spinal cord was detached from the body and fixed in $4 \%$ paraformaldehyde in PBS for $1 \mathrm{~h}$ at room temperature, after which the tissue was spread open to produce flat-mount preparations (Avraham et al., 2009, 2010).

Immunohistochemistry. Embryos were fixed overnight at $4^{\circ} \mathrm{C}$ in $4 \%$ paraformaldehyde/0.1 M phosphate buffer, washed twice with PBS, incubated in $30 \%$ sucrose/PBS for $24 \mathrm{~h}$, and embedded in OCT. Cryostat sections $(14 \mu \mathrm{m})$ were collected on Superfrost Plus slides and kept at $-70^{\circ} \mathrm{C}$. The following antibodies were used: rabbit polyclonal GFP antibody (Invitrogen); Pax2 (Abcam), myc (9E10), Isl1 (4D5), Lhx1/5 (4F2), and Lhx2/9 (rabbit serum) (all provided by T. Jessell, Columbia University, New York, NY); Axonin/TAG-1 (23.4-5 from the Hybridoma Bank); and Lhx9 (Santa Cruz sc-19348). Cy2, RRX, and Cy5 were used as fluorochromes. Images were taken under a microscope (Axioscope 2; Zeiss) with a digital camera (DP70; Olympus) or confocal microscope (FV1000; Olympus).

$D N A$. The chick Isll gene was obtained from Artur Kania (Institut de recherches cliniques de Montréal, Quebec, Canada). The 215 enhancer element was amplified by PCR from genomic mouse DNA using the following primers: 5 '-ATGAGCTCCATCTGCACAAAGGTTGGGAGG and $3^{\prime}$-ATGCTAGCGCCACCCTCTTCTCCATTTCC, and cloned into the SacI-NheI sites of the appropriate Cre and Gal4 plasmids. The 242 and 586 human enhancer elements were obtained from the Enhancer Browser project (http://enhancer.lbl.gov/). The enhancers were amplified by PCR using the following primers: 5'-ATGAGCTCTACAAAAAAGCAGGCTCCGC and 3 '-GCTTAATTAATACAAGAAAGCTGGGTCGGC, and cloned into the SacI-PacI sites of the appropriate Cre and Gal4 plasmids.

\section{Results}

\section{Characterization of dI3 enhancer elements}

dI3 are excitatory interneurons that are believed to relay nociceptive sensory information (Xu et al., 2008). They are characterized by the combinatorial expression of a number of transcription factors: Tlx3, Prrxl1, Brn3a, Olig3, Ascl1, and Isl1 (Helms and Johnson, 2003; Xu et al., 2008). The expression of Isl1 in spinal interneurons is restricted to $\mathrm{dI} 3$, while the other transcription factors are expressed in additional interneurons. Hence, to follow the axonal trajectory pattern of dI3 neurons, conserved noncoding sequences flanking the Isl1 gene were screened. Five human genomic elements that are evolutionally conserved $(215,543$, 586,1321 , and 1419) and yield LacZ expression in Isl1-expressing neurons (motor neurons and DRG) in E11.5 transgenic mice were characterized by the Enhancer Browser project (Visel et al., 2007a,b) (http://enhancer.lbl.gov/). In two of them, 215 and 586, expression of LacZ in the dorsal spinal cord was apparent. Both elements are located on human chromosome 5 between Isl1 and PARP8, where 215 is located 342,626 bp and 586 is located 196,791 bp $3^{\prime}$ to the Isl1 gene. A third element, 242, located at human chromosome 2 in an intron of the gene, a member of the MAPKKK family of signal transduction molecules, was also selected.

The three elements were cloned downstream to the Cre recombinase and electroporated along with a conditional, Cre-dependent nGFP into the chick neural tube following a procedure described previously (Zisman et al., 2007; Avraham et al., 2009, 2010). 215 directed nGFP expression in motor neurons (MNs), dI3 neurons, roof plate (RP) cells, and DRG neurons (Fig. $1 A, B, H$; supplemental Fig. S1, available at www.jneurosci.org as supplemental material). 586 directed expression in MNs and dI3 and dI2 neurons (Fig. 1C,H ). 242 directed expression in MNs and dI3, dI2, and dI1 neurons (Fig. $1 D, E, H$ ). The refinement of expression to $\mathrm{dI} 3$ neurons was attained by intersection between the 242 and the 215 enhancer elements (Avraham et al., 2009). GFP expressed under a dual conditional cassette is expressed exclusively in Isl $1^{+}$spinal neurons (dI3-74 $\pm 26 \%, \mathrm{MN}-22 \pm 27 \%$ ) (Fig. $1 \mathrm{~F}-\mathrm{H}$ ). No expression was evident in dI1, dI2, or DRG neurons or roof plate cells (Fig. $1 F-H$; supplemental Fig. S1, available at www.jneurosci.org as supplemental material). Thus, the combinatorial usage of the 215 and 242 enhancers (herein indicated as EdI3) is appropriate for labeling dI3 neurons and axons.

\section{The axonal projection pattern of $\mathrm{dI} 3$ neurons}

The axonal projection pattern of $\mathrm{dI} 3$ neurons within the neural tube was studied at E6 using an open-book preparation of electroporated neural tubes. The neural tubes of six embryos, labeled with GFP under the control of EdI3, were analyzed and yielded similar axonal patterns. At E6 dI3 neurons are constrained to the ventral margin of the dorsal-half neural tube (Fig. $2 A-C$, arrowheads). Their axons form two longitudinal fascicules at the ipsilateral neural tube: a broad fascicule that encompasses the ventral third neural tube (Fig. 2A-C, yellow arrows) overlapping the location of the VLF; and a tight fascicule along the ipsilateral side the dorsal midline (Fig. $2 A-C$, white arrows), overlapping the DF. At the sacral level, at either the dorsal or ventral fascicules, axons are present caudally to the electroporated cell bodies (Fig. $2 A, C)$. Thus, the sacral dI 3 neurons project their axons caudally. In agreement, the growth cones of the sacral and the lumbar dI 3 neurons at the VLF point caudally (supplemental Fig. S2 $A^{\prime}-D^{\prime}$, available at www.jneurosci.org as supplemental material). At the thoracic level, the growth cones of the VLF fascicule point rostrally (supplemental Fig. S2 $A-D$, available at www.jneurosci.org as supplemental material), implying that the rostral $\mathrm{dI} 3$ neurons turn rostrally. The tightness of the dorsal fascicule precludes the identification of growth cones. However, a neuron projecting dorsally and rostrally is observed at high magnification of a sparsely electroporated neural tube (Fig. 2D).

The large width of the VLF bundle may arise from axons turning longitudinally at different dorsoventral locations, or axons may deflect dorsally or ventrally within the longitudinal plane. The abundance of axons at the VLF obstructs the exact position of the transverse to longitudinal turn. However, axons deflecting dorsally from the ventral boundary of the VLF are seen at the margins of the electroporated zone (sacral and 

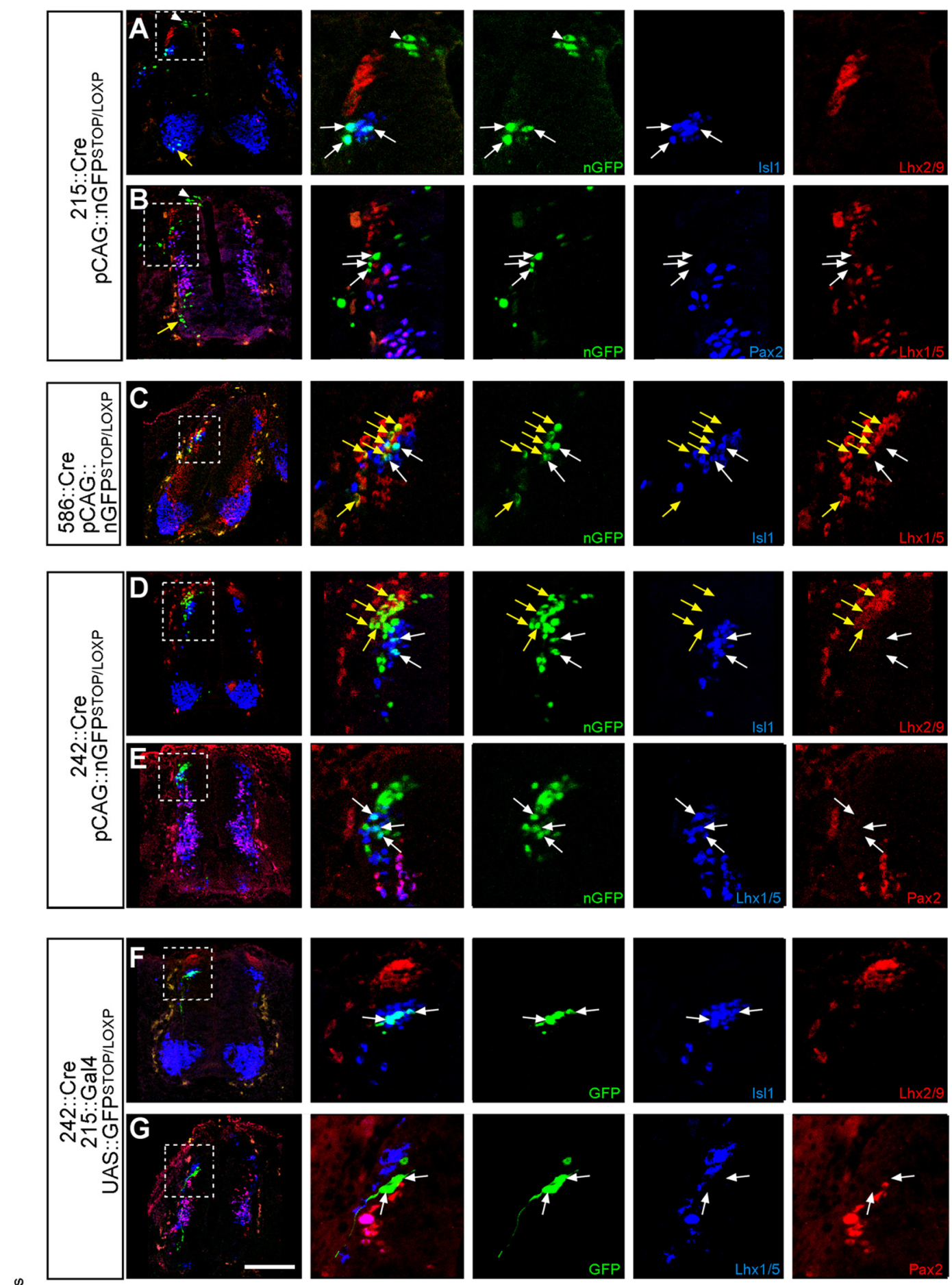

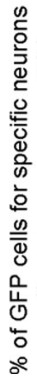

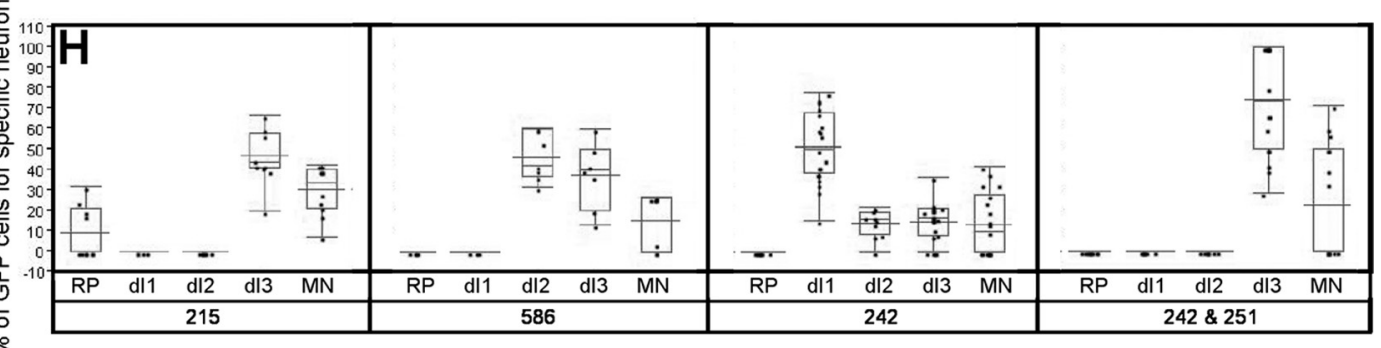

Figure 1. Characterization of d II3 enhancers. 215, 586, and 242 enhancer elements were cloned upstream to Cre recombinase and electroporated with a conditional nGFP (CAGG-loxPSTOP-loxP-nGFP) (A-E). 215 enhancer was cloned upstream to Gal4 and electroporated with 242:::Cre and a double-conditional GFP plasmid (UAS::IoxP-STOP-loxP-GFP) (F, G). Chick embryos were electroporated at stage 16 and fixed at E4. Cross sections of electroporated neural tube were stained with interneuron-specific antibodies. The images on the left show the entire neural tube and the adjacent images are high magnifications of the boxed areas, which show the dorsal neural tube stained with the indicated antibodies. (Figure legend continues.) 
cervical levels), where fewer neurons are labeled (supplemental Fig. S3, available at www.jneurosci.org as supplemental material). At the sacral levels, dI3 axons are deflected caudally and dorsally (supplemental Fig. S3A-C, available at www.jneurosci. org as supplemental material) and at the cervical level rostrally and dorsally (supplemental Fig. S3D-F, available at www. jneurosci.org as supplemental material). Thus, the ventrally projecting dI3 axons turn from the transverse to the longitudinal plane at the ventral margin of the VLF and subsequently curve dorsally within the VLF (Fig. 2E).

Only a few (less then $2 \%$ ) axons are visible at the contralateral side (Fig. $2 A$; see Fig. $8 H$ ). Hence, dI3 neurons are subdivided into four neuronal populations: $\mathrm{dI} 3_{\mathrm{dorsal} / \text { caudal }}$ and $\mathrm{dI} 3_{\mathrm{dorsal} / \mathrm{rostral}}$, which project their axons toward the roof plate and turn longitudinally at the DF, and $\mathrm{dI} 3_{\text {ventral/caudal }}$ and $\mathrm{dII} 3_{\text {ventral/rostral }}$, which project ventrally and turn longitudinally at the VLF (Fig. 2E).

Other ipsilaterally projecting interneurons, $\mathrm{dI} 1_{\text {ipsi }}$ (Wilson et al., 2008; Avraham et al., 2009) and V1 (Alvarez et al., 2005), project their axons laterally toward the marginal zone. The relatively large gap between the cell bodies and the VLF fascicule of dI3 neurons (Fig. 2) suggests that $\mathrm{dI} 3_{\text {ventral }}$ axons initially extend ventrally, before turning laterally to the longitudinal plane. To map the longitudinal turning point, cross sections of EdI3::GFP electroporated neural tubes were inspected (Fig. 3; supplemental Figs. S4, S5, available at www.jneurosci.org as supplemental material). Motor and DRG axons were costained with axonalspecific antibodies to BEN (SC1/DM-GRASP protein) that label motor and DRG axons and the floor plate, and anti-axonin-1 MAB that labels DRG neurons. $\mathrm{dI} 3_{\text {ventral }}$ axons are initially projected ventrally. They even turn diagonally toward the ventral midline (Fig. $3 A, A^{\prime}$, white arrows; supplemental Fig. S4, available at www.jneurosci.org as supplemental material). As they encounter the motor neuron domain (labeled with the BEN antibody), they turn laterally and elongate together with the axons of the motor neurons (Fig. $3 A, A^{\prime}$, yellow arrows; supplemental Fig. S4 $A-E$, available at www.jneurosci.org as supplemental material). At the marginal zone, $\mathrm{dI} 3_{\text {ventral }}$ axons turn longitudinally, as evidenced by the punctuated staining of axons in the white matter (Fig. $3 A, A^{\prime}$, arrowheads; supplemental Fig. S $4 A-E$, available at www. jneurosci.org as supplemental material). At E6, the longitudinally projecting $\mathrm{dI} 3_{\text {ventral }}$ axons overlap with the exit point of motor neurons (Fig. $3 B, B^{\prime}$; supplemental Fig. $S 4 F-I$, available at www. jneurosci.org as supplemental material). The confinement of the longitudinally projecting $\mathrm{dI} 3_{\text {ventral }}$ axons at the VLF to the motor axons zone is also illustrated in a costained open-book preparation. The dI 3 axons are restricted to the motor axon (BEN-positive) territory (Fig. $3 C-C^{\prime \prime}$, yellow arrows).

Thus, $\mathrm{dI} 3_{\text {ventral }}$ axons make two abrupt $\left(\sim 90^{\circ}\right)$ turns: a turn at the transverse plane from ventral-to-lateral projection, and a turn at the marginal zone, from the transverse to the longitudinal plane. At the ventral-to-lateral turn, dI $3_{\text {ventral }}$ axons seem to follow the route of the motor axons, and at the transverse-to-

\footnotetext{
(Figure legend continued.) $\ln A$ and $B$, the yellow arrows point to $M N s$, the arrowheads to roof plate cells, and the white arrow to $\mathrm{dl} 3 \mathrm{n}$ neurons. In $C$, the yellow arrows point to $\mathrm{dl} 2$ neurons and the white arrows to $\mathrm{dl} 3$ neurons. In $\boldsymbol{D}$, the yellow arrows point to dl1 neurons and the white arrows to $\mathrm{d} \mid 3$ neurons. In $\boldsymbol{E}$, the white arrows point to $\mathrm{d} \mid 2$ neurons. In $\boldsymbol{F}$ and $\boldsymbol{G}$, the white arrows point to $\mathrm{dl} 3$ neurons. The ratio of labeled interneurons to the total GFP-positive cells for each enhancer and enhancer combination is indicated in $\boldsymbol{H}$. MNs are the ventral Isl1 ${ }^{+}$cells. $\mathrm{dl} 3$ are the dorsal $\mathrm{s} \mid 1^{+}$cells. dl 2 are the $\mathrm{Lhx} 1 / 5^{+} / \mathrm{Pax} 2^{-}$cells, dl1 are the Lhx2/9 ${ }^{+}$cells, and RP cells are the dorsal midline cells. Scale bar in $\mathbf{G}$ is $250 \mu \mathrm{m}$ for images of the entire neural tube (left side) and $50 \mu \mathrm{m}$ for the magnifications.
}

longitudinal turn, $\mathrm{dI} 3_{\text {ventral }}$ axons "depart" from the axonal tracks of the motor neurons. Thus, motor axons and marginal zone-derived cues may control the "association and separation" of $\mathrm{dI} 3_{\text {ventral }}$ and motor axons, respectively.

For viewing the axonal pathway of $\mathrm{dI} 3_{\text {dorsal }}$ neurons, cross sections of electroporated embryo were stained with the antiaxonin antibody that labels the axons of DRG neurons. $\mathrm{dI} 3_{\text {dorsal }}$ axons are projected dorsally toward the DREZ. In cross sections, punctuated GFP staining is evidenced at the DREZ (Fig. $3 D, D^{\prime}$, white arrows; supplemental Fig. S5, available at www.jneurosci. org as supplemental material). In open-book preparations, $\mathrm{dI} 3_{\text {dorsal }}$ axons are intermingled with the longitudinally projecting DRG axons (Fig. 3C, white arrows). Thus, $\mathrm{dI} 3_{\text {dorsal }}$ axons turn longitudinally at the DREZ. The longitudinal fascicule of $\mathrm{dI} 3_{\text {dorsal }}$ neurons is intermingled with the longitudinally projecting DRG axons at the dorsal funiculus (Fig. 3E).

Interestingly, the axons of $\mathrm{dI} 3$ neurons that express Isl1 are associated with axons of either motor or DRG neurons that also express Isl1 (Fig. 3E). Thus, an Isl1-mediated homophilic interaction may control the ventral-to-lateral turn of $\mathrm{dI} 3_{\text {ventral }}$ axons, and the longitudinal turn of $\mathrm{dI} 3_{\text {dorsal }}$ axons.

\section{The longitudinal axonal fascicules of dI3 do not intermingle with other dorsal interneurons}

While dorsal interneurons differentiate, their soma position, from dorsal to ventral, is arranged in the following order: dI1, dI2, and dI3. At E5-E6, as they assume ventral migration, the dorsal/ventral boundaries between them are disrupted, and their soma positions are more mixed (Lee and Jessell, 1999; Helms and Johnson, 2003). Despite the bias toward cell intermingling, their axons form an interneuron-specific bundle as they project longitudinally. We have demonstrated that the longitudinal fascicules of $\mathrm{dI} 1$ and $\mathrm{dI} 2$ axons are arranged in homotypic bundles, in which the dI2 fascicule is positioned dorsally to the dI1 bundle (Avraham et al., 2009). To learn whether $\mathrm{dI} 3$ axon longitudinal tracks are also segregated, we focused on the relative arrangement of $\mathrm{dI} 1$ and $\mathrm{dI} 3$ axons.

For open-book preparations (two open books), GFP and taumyc differential labeling of dI3 and dI1 axons was attained using the Gal4/UAS system for dI3 neurons and the Cre/LoxP system for dI1 neurons. The dI1-specific enhancer element EdI1 (Avraham et al., 2009) and the 215 enhancer element were used to label dI 1 and $\mathrm{dI} 3$ neurons, respectively (Fig. $4 A, A^{\prime}$ ). To eliminate labeling of motor neurons, the electroporation was aimed at the dorsal neural tube. At the contralateral side of an E6 open-book preparation, mostly dI $1^{\text {taumyc }}$ axons are visible (Fig. $4 A$ ). At the ipsilateral side, a longitudinal fascicule of dII ${ }^{\text {taumyc }}$ is positioned between the DF and VLF longitudinal dI $3{ }^{\mathrm{GFP}}$ fascicules (Fig. $4 \mathrm{~A}$ ). However, a few $\mathrm{dI} 3_{\text {ventral }}^{\mathrm{GFP}}$ axons are detected within the dI $1^{\text {taumyc }}$ bundle (Fig. $4 A^{\prime}$ ). These axons may still be segregated from dI 1 axons along the medial-lateral axis.

To gain more insight into the relative order of the longitudinal $\mathrm{dI} 1$ and $\mathrm{dI} 3_{\text {ventral }}$ fascicules, cross sections of double-labeled neural tubes were inspected. Since the exit point of motor axons and the longitudinal turning point of $\mathrm{dI} 3_{\text {ventral }}$ axons overlap along the medial-lateral axis, dI3-specific expression should be managed. To achieve this goal, taumyc was expressed in dI1 neurons using the EdI1 enhancer element and the PhiC31o/Att sitespecific recombination system (Raymond and Soriano, 2007), together with the EdI3::GFP plasmid combination (Fig. 4B). Segregation of the longitudinal projection of $\mathrm{dI} 1$ and $\mathrm{dI} 3$ axons (punctuating the white matter) along the dorsal-ventral and medial-lateral axes is evident in cross section of E6 embryos. In the small region in which $\mathrm{dI} 1$ and $\mathrm{dI} 3$ axons are at the same D/V level, 
dI1 axons are positioned medially to dI1 axons (Fig. $4 \mathrm{~B}$ ). The exclusion of the longitudinal axonal fascicules of the dorsal dI1-3 neurons (Fig. 4C) may represent an example of a more general phenomenon, in which all the spinal interneurons project their axons in the white matter in fascicules at defined IN-specific dorsal/ ventral and lateral/medial coordinates.

\section{Isl1, Lhx1, and Lhx9 cross-repress each other}

In motor neurons, reciprocal crossrepression between the Lim-HD proteins Lhx1 and Isl1 ensures a sharp boundary between the LMCl and LMCm subpopulations and instructs axonal choice between the dorsal and ventral limbs, respectively (Kania and Jessell, 2003). Similarly, reciprocal cross-repression between Lhx9 and Lhx1 governs the rostral versus caudal turning of the longitudinally projecting $\mathrm{dI} 1$ and $\mathrm{dI} 2 \mathrm{ax}-$ ons (Avraham et al., 2009). In both cases, Lim-HD proteins are not required for neuronal cell fate (Kania and Jessell, 2003; Pillai et al., 2007; Luria et al., 2008; Wilson et al., 2008). We examined whether Isll plays a similar role in the fate and axonal patterning of dI3 neurons.

We initially tested whether Isll and the neighboring Lim-HD proteins, Lhx $2 / 9$ expressed in dI1 neurons and Lhx1/5 expressed in $\mathrm{dI} 2$, dI4, and dI6, cross-repress each other. The ratio of neurons coexpressing the ectopic Lim-HD protein and the endogenous Lim-HD protein at the electroporated side versus the number of Lim-HD cells at the control side was calculated. Electroporation was performed at $\mathrm{HH}$ stages 14 and 19. At stage HH14 (referred to as early), most of the interneurons are premitotic, while at HH19 (referred to as late), most of the interneurons are postmitotic. In neural tubes electroporated with nGFP, $57.6 \%$ of the electroporated dI 1 neurons coexpressed nGFP and Lhx2/9, 35\% of Lhx1/5 neurons coexpressed nGFP and Lhx $1 / 5$, and $63 \%$ of dI 3 neurons coexpressed nGFP and Isl1 (Fig. 5E-H). Ectopic expression of Lhx9, in either early or late dI3 neurons, resulted in substan-

tial reduction of neurons coexpressing Lhx9 and Isl1 (Fig. 5C): at $\mathrm{HH} 14,5.5 \%$, and at $\mathrm{HH} 19,4.24 \%$ (Fig. 5E). Likewise, Isl1 affected a comparable decrease in the expression of Lhx9 proteins (Fig. 5A). Ectopic Isl1 resulted in $9.9 \%$ and $8.1 \%$ of neurons coexpressing Lhx 9 together with Isl 1 at either early or late electroporated neural tubes, respectively (Fig. 5F). Similar reciprocal cross-repression was scored using Lhxl and Isl1 (Fig. $5 B, D$ ). In neural tubes electroporated with Lhx1, 20.4\% of the early electroporated and $12.9 \%$ of the late electroporated cells coexpressed Lhx 1 and Isl1 (Fig. 5G). In neural tubes electroporated with Isl1, 9.2\% of the early electroporated and

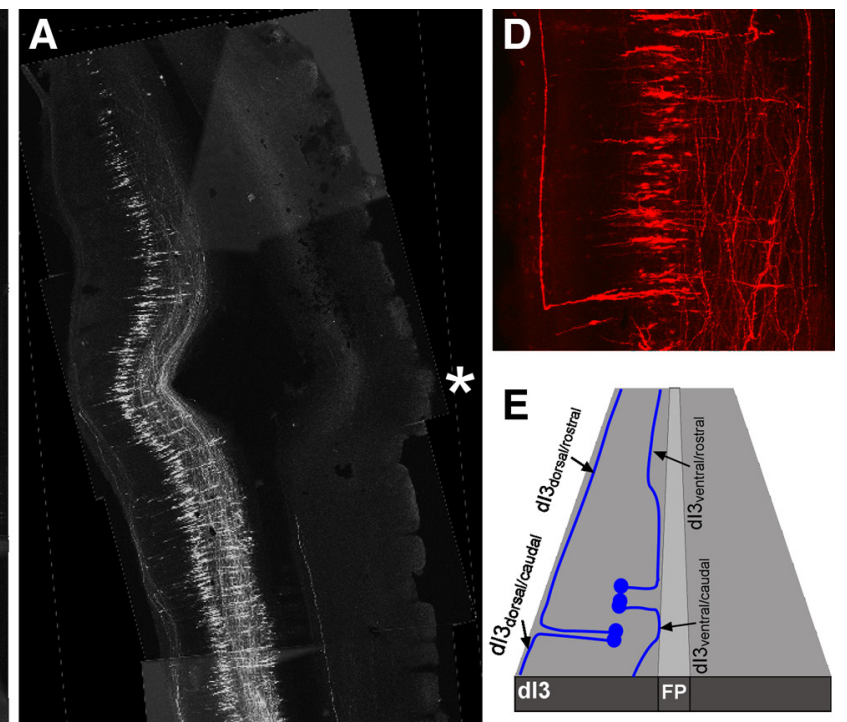

Figure 2. Axonal projection pattern of dl3 neurons. Chickembryos were electroporated at stage 16 (left side) with Edl3 enhancers along with a Cre/Gal4GFP plasmid (215::Gal4 + 242::Cre + UAS-LoxP-STOP-LoxP-GFP). Spinal cords were removed at E6, fixed, and analyzed as open-book preparations. Whole neural tubes (sacral to cervical) are presented $(A)$. Confocal images were taken and photomerged using Photoshop software. High-power magnification of the thoracic ( $\boldsymbol{B}$ ) and sacral ( $\boldsymbol{C}$ levels are shown. A single dl3 neuron projecting toward the dorsal midline and turning rostrally is shown in $\boldsymbol{D}$. The scheme in $\boldsymbol{E}$ illustrates the axonal projection pattern of the dI3 neuronal ventral longitudinal fascicule at the VLF. The white arrows point to the dorsal longitudinal fascicule at the DF. The asterisk represents the level of the limbs. FP, Floor plate. Scale bar is $300 \mu \mathrm{m}$ for $\boldsymbol{A}, 200 \mu \mathrm{m}$ for $\boldsymbol{B}$ and $\boldsymbol{C}$, and $150 \mu \mathrm{m}$ for $\boldsymbol{D}$.

$13.8 \%$ of the late electroporated cells coexpressed $\mathrm{Lhx} 1 / 5$ and Isl1 (Fig. 5H).

Cross-repression may arise from a change of cell fate. Thus, ectopic expression of Isl1 may determine $\mathrm{dI} 3$ fate, which, as a consequence, will lead to downregulation of the reciprocal Lim-HD protein. The expression of dI cell fate markers was studied following ectopic expression of Isl1. The expression pattern of Brn3a (expressed in dI1,2,3,5) (Fig. 6A), Pax2 (dI4,6) (Fig. 6B), and Tlx3 (dI3,5) (Fig. 6C) was not altered following Isl1 ectopic expression. Therefore, Isl1 is not sufficient to suppress the cell fate of other interneurons or to induce ectopic dI3 cell fate. 

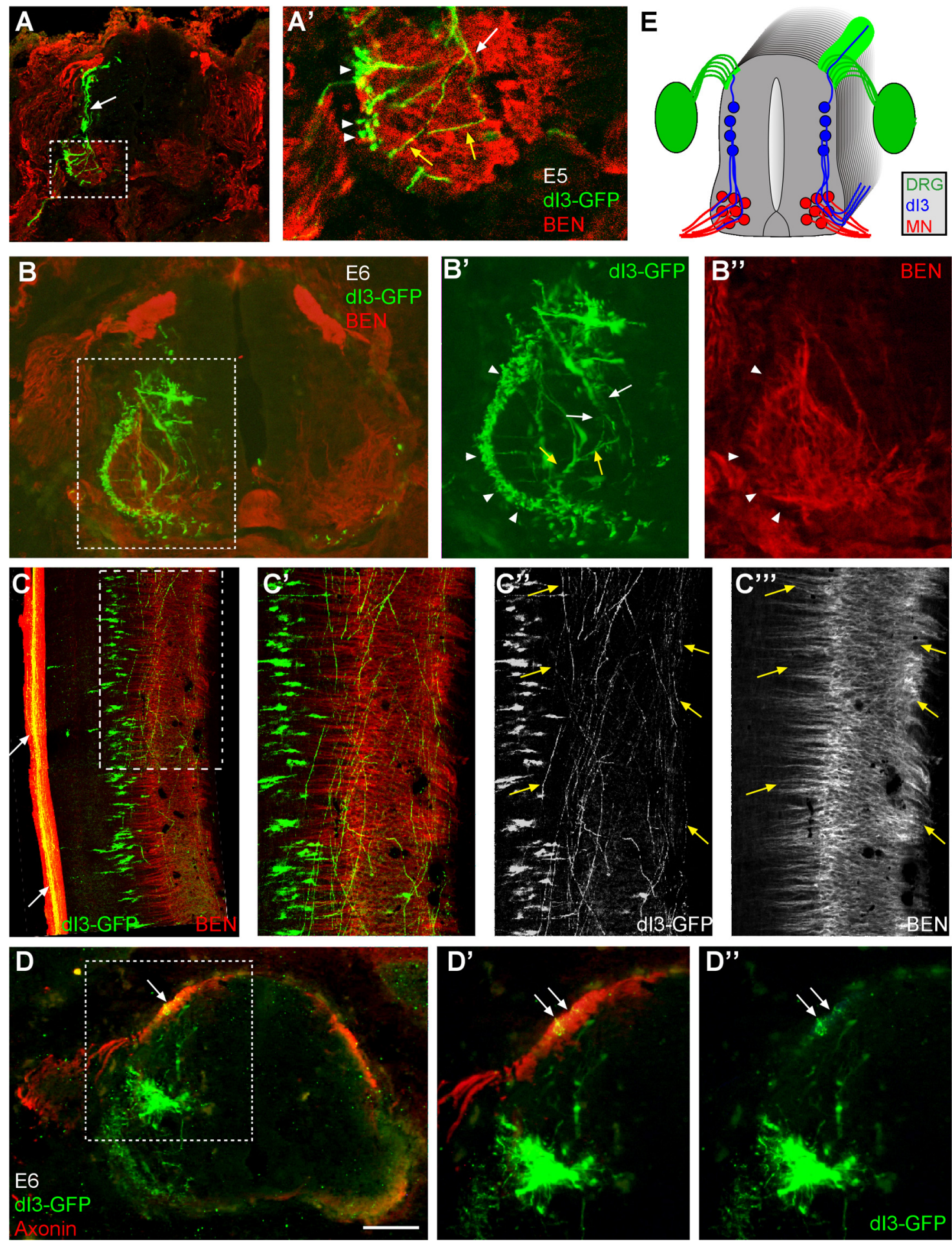

Figure 3. dl3 axons turn along the axons of motor and DRG neurons. Chick embryos were electroporated as in Figure 2 and analyzed in cross sections at E5 (A) and E6 (B-D). The BEN antibody, which labels motor axons, floor plate cells, and DRG axons, was used to label motor axons ( $A-C$. Anti-axonin antibody was used to label the axons of DRG neurons and the DREZ (D). Aschemedemonstrating the axonal turning points of dl 3 axons is presented in $\boldsymbol{E}$. High-power magnifications of the boxed regions in $\boldsymbol{A}-\boldsymbol{D}$ are shown in $\boldsymbol{A}^{\prime}, \boldsymbol{B}^{\prime}, \boldsymbol{B}^{\prime \prime}, \boldsymbol{C}^{\prime}, \boldsymbol{C}^{\prime \prime}, \boldsymbol{C}^{\prime \prime}, \mathbf{D}^{\prime}$, and $\boldsymbol{D}^{\prime \prime}$. In $\boldsymbol{A}$ and $\boldsymbol{B}$, the white arrows point to the initial dorsoventral projection of $\mathrm{dl} 3_{\text {ventral }}$ axons. The yellow arrows point to the medial-to-lateral turn of $\mathrm{dl} 3_{\text {ventral }}$ axons. The arrowheads point to the longitudinal turn of $\mathrm{dl} 3_{\text {ventral }}$ axons. In $C$, the yellow arrows point to $\mathrm{dl} 3_{\text {ventral }}$ axons at the dorsal and ventral margins of the VLF and the white arrows point to $\mathrm{dl}_{3}{ }_{\text {dorsal }}$ axons within the afferent fascicule at the $D F$. In $\boldsymbol{D}$, the white arrows point to the longitudinal turn of $\mathrm{d} \mid 3_{\text {dorsal }}$ axons. Scale bar in $\boldsymbol{D}$ is $300 \mu \mathrm{m}$ for $\boldsymbol{A}, 100 \mu \mathrm{m}$ for $\boldsymbol{A}^{\prime}, \boldsymbol{B}^{\prime}$, and $\boldsymbol{B}^{\prime \prime}, 150 \mu \mathrm{m}$ for $\boldsymbol{B}, 150 \mu \mathrm{m}$ for $\boldsymbol{C}, 75 \mu \mathrm{m}$ for $\boldsymbol{C}^{\prime}, \boldsymbol{C}^{\prime \prime}$, and $\boldsymbol{C}^{\prime \prime}, 170 \mu \mathrm{m}$ for $\boldsymbol{D}$, and $75 \mu \mathrm{m}$ for $\boldsymbol{D}^{\prime}$ and $\boldsymbol{D}^{\prime \prime}$.

Isl1 is not required for the acquisition $\mathrm{dI} 3$ cell fate

The fate of dI3 neurons that do not express Isl1 was studied in Isl1 hypomorph mice (Isllhypo) that contain a PGK-Neo cassette within an intron, resulting in reduction of Isl1 expression levels (Sun et al., 2008; Song et al., 2009). Isl1hypo mice express low levels of Isl1 in motor neurons and lack expression of Isl1 in dI3 neurons (Fig. $7 B, B^{\prime}, G, G^{\prime}$ ). The pattern of expression of the cell fate markers, Brn3a expressed in dI1,2,3,5 neurons (Fig. $7 A, A^{\prime}, F, F^{\prime}$ ), Tlx3 expressed in dI3,5 neurons (Fig. $7 A-C, A^{\prime}-$ $C^{\prime}, F-H, F^{\prime}-H^{\prime}$ ), Lhx1/5 expressed in dI2,4,6 neurons (Fig. 
$\left.7 C, C^{\prime}, H, H^{\prime}\right)$, Pax2 expressed in dI4,6 neurons (Fig. $7 D, D^{\prime}, I, I^{\prime}$ ), and Lhx9 expressed in dI1 neurons (Fig. $7 E, E^{\prime}, J, J^{\prime}$ ), was studied in cross sections of E11.5 Isllhypo and littermate controls. The expression pattern of all the above transcription factors is indistinguishable between the Isllhypo and wild-type mice (Fig. 7). Hence, Isl1, like Lhx2/9 in dI1 (Wilson et al., 2008) and Lhx1/5 in dI2 (Pillai et al., 2007), is not required for the acquisition of $\mathrm{dI} 3$ cell fate.

\section{Ectopic Isl1 confers dI3 axonal patterning to dI1 neurons}

We have shown previously that reciprocal replacement of the Lim-HD code in dI 1 and dI2 neurons, where dI1 neurons express Lhxl and dI2 neurons express Lhx9, is sufficient to confer characteristic dI 1 axonal pathways to $\mathrm{dI} 2^{\mathrm{Lhx} 9}$, and characteristic dI2 axonal pathways to $\mathrm{dI} 1^{\mathrm{Lhx} 1}$ (Avraham et al., 2009). To test whether Isl1 is involved in patterning the axonal trajectories of dI3 neurons, it was expressed ectopically in dI1 neurons (EdI1::Cre + CAGG-LoxPSTOP-LoxP-Isl1-IRES-taumyc).

The axonal trajectories of $\mathrm{dI} 1$ and $\mathrm{dI} 3$ neurons differ from each other in several aspects (Fig. $8 A, B, D, F, I$ ): (1) dI 3 are ipsilaterally projecting neurons, while dI1 are subdivided into ipsilaterally $\left(\mathrm{dI} 1_{\text {ipsi }}\right)$ and contralaterally ( $\left.\mathrm{dI}_{\text {comm }}\right)$ projecting neurons; (2) a subpopulation of $\mathrm{dI} 3$ neurons $\left(\mathrm{dI} 3_{\text {dorsal }}\right)$ project their axons dorsally, while none of the dI1 subpopulations send axons dorsally; (3) dI $1_{\mathrm{ipsi}}$ axons turn laterally toward the marginal zone, while $\mathrm{dI} 3_{\text {ventral }}$ axons initiate ventral projection; and (4) $\mathrm{dI} 1_{\mathrm{ipsi}}$ axons turn laterally, dorsally to the motor neurons, while $\mathrm{dI} 3_{\text {ventral }}$ axons turn laterally within the motor neurons.

The fate of dI $1^{\text {Isl1 }}$ axons was studied in E6 open-book preparation and cross sections from E5 and E6 electroporated embryos (six embryos for each). To score the ipsilateral versus contralateral choice, the number of axons in the longitudinal fascicules of open-book preparations was measured. In $\mathrm{dI} 3{ }^{\mathrm{GFP}}$ axons, $<2 \%$ of the axons crossed the floor plate, and elongated at the contralateral side (Fig. $8 \mathrm{~B}, \mathrm{H}$ ). Twenty percent of $\mathrm{dI} 1{ }^{\mathrm{GFP}}$ axons crossed the midline (Fig. $8 A, H$ ). However, only $10 \%$ of the dII $1^{\text {Isl1/taumyc }}$ neurons crossed the midline (Fig. 8C,H). Isll thus causes an ipsilateral bias to the $\mathrm{dI} 1_{\text {comm }}$ neurons.

The feature of the contralateral-to-ipsilateral change in axonal choice was studied in cross sections of electroporated embryos (Fig. $8 D-G$ ). At E5, most of the dI1 axon-projecting neurons are of the $\mathrm{dI} 1_{\text {comm }}$ subpopulation, which send their axons toward and across the floor plate (Fig. 8D). At a comparable develop-
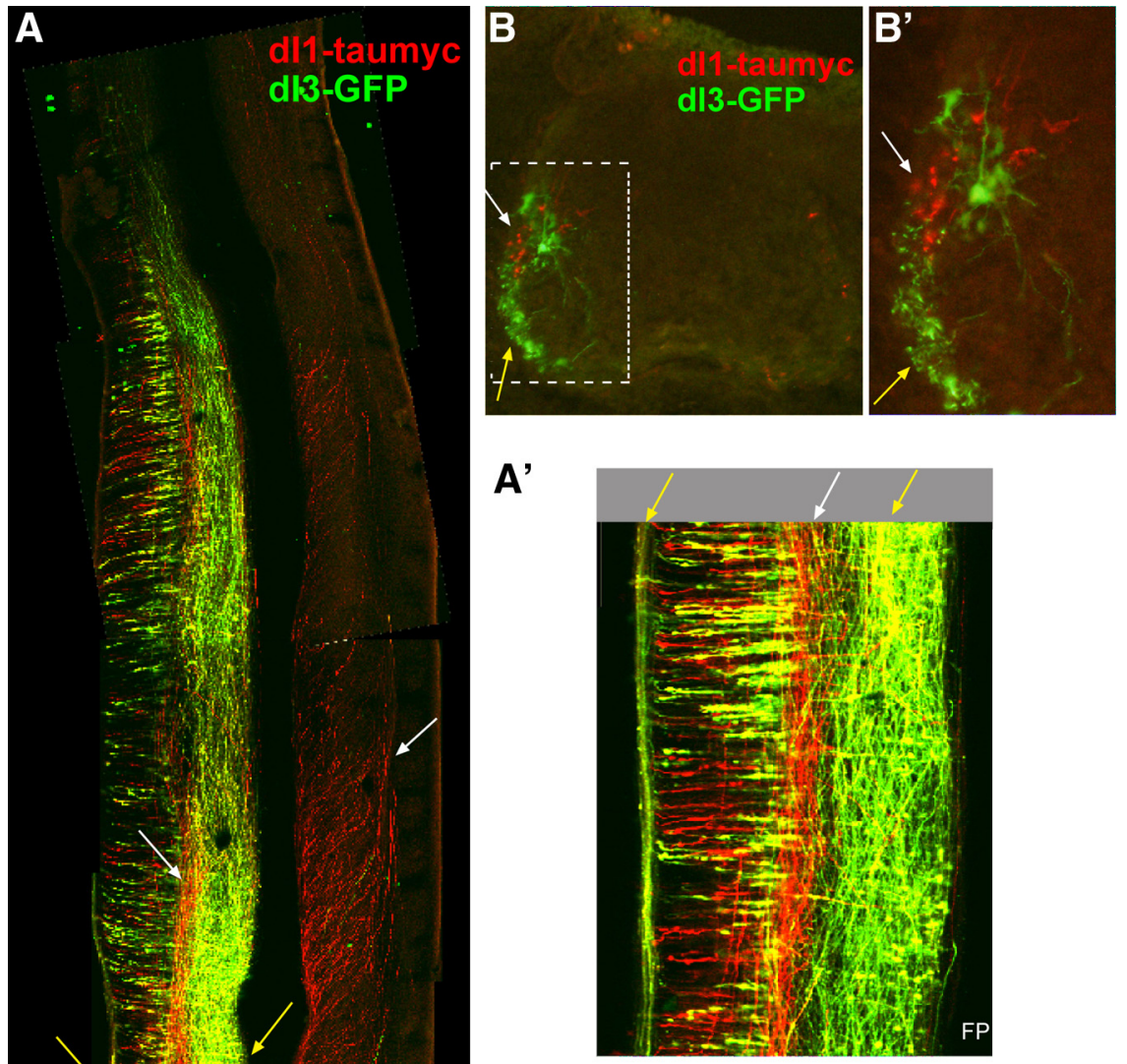

Figure 4. Double labeling of $\mathrm{dl} 1$ and $\mathrm{dl} 3$ axons reveals the topographic arrangement of the dorsal IN. Plasmid combinations that differentially label dl1 and dI3 neurons were used: $215:: G a l 4+$ UAS::GFP and Edl1::Cre + pCAGG-LoxP-STOP-LoxP-taumyc for $\boldsymbol{A}$; and 215::Gal4 + 242::Cre + UAS::LoxP-STOP-LoxP-GFP and Ed11::PhiC310 + pCAGG-AttB-STOP-AttP-taumyc for $\boldsymbol{B}$. Magnification of the boxed areas in $\boldsymbol{A}$ and $\boldsymbol{B}$ are shown in $\boldsymbol{A}^{\prime}$ and $\boldsymbol{B}^{\prime}$. Yellow arrows point to the longitudinal fascicules of dl 3 axons. White arrows point to the longitudinal fascicules of $\mathrm{dl} 1$ axons. A scheme that demonstrates the location of the dl1-3 neurons and axons is shown in $\boldsymbol{C}$. FP, Floor plate. Scale bar is $300 \mu \mathrm{m}$ for $\boldsymbol{A}, 200 \mu \mathrm{m}$ for $\boldsymbol{A}^{\prime}$ and $\boldsymbol{B}$, and $100 \mu \mathrm{m}$ for $\boldsymbol{B}^{\prime}$.

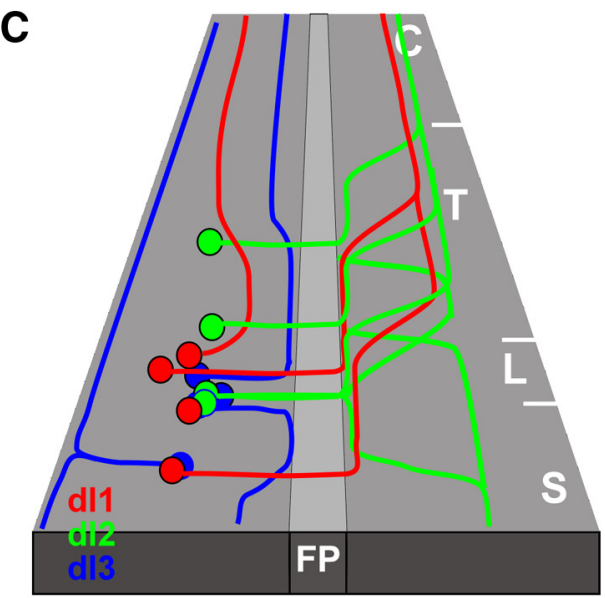

mental stage, dII ${ }^{\text {Isl1/taumyc }}$ axons start to project toward the floor plate. However, as with $\mathrm{dI} 3_{\text {ventral }}$ axons, they turn laterally and extend with the motor axons as they encounter the motor neuron zone (Fig. $\left.8 E, E^{\prime}, E^{\prime \prime}\right)$. At the marginal zone, similar to $\mathrm{dI} 3_{\text {ventral, }}$ $\mathrm{dI} 1^{\text {Isl1/taumyc }}$ axons turn longitudinally, as evidenced by the punctuated staining in the white matter (Fig. $8 E, E^{\prime}, E^{\prime \prime}$, arrowheads). The axons of $\mathrm{dI} 1_{\mathrm{ipsi}}$ neurons are evident at E6. $\mathrm{dI} 1_{\mathrm{ipsi}}$ axons are 

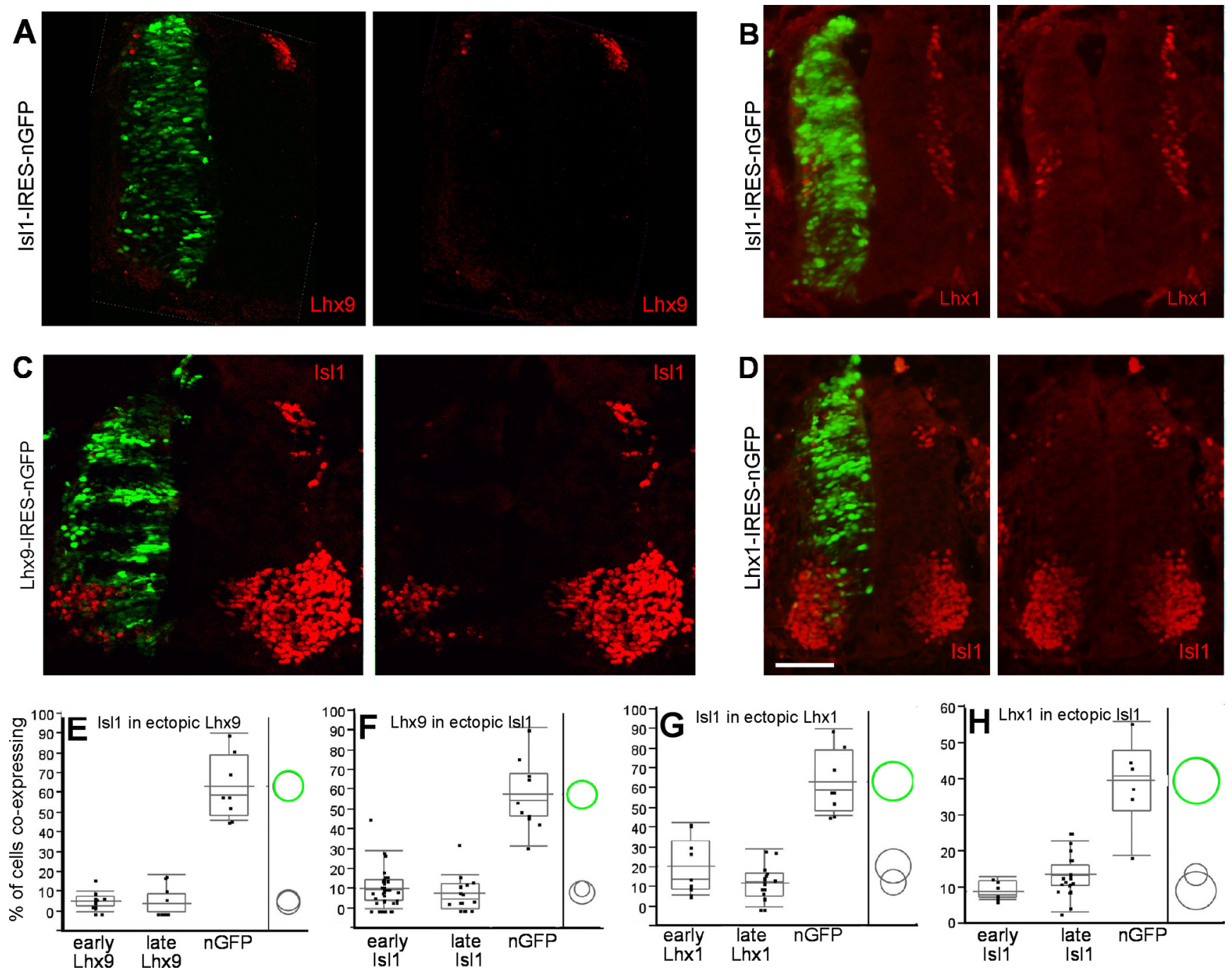

Figure 5. Is 1 represses $\operatorname{Lhx} 1 / 5$ and Lhx2/9 without altering IN cell fate. $A, C,|s| 1$ and Lhx 9 cross-repress each other. $B, D,|s| 1$ and Lhx 1 cross-repress each other. The images on the left show overlapping between electroporated cells and Lim-HD-positive neurons. The images on the right show the Lim-HD channel. $\boldsymbol{E}-\boldsymbol{H}$, For quantification, the number of cells coexpressing the electroporated gene and the endogenous Lim-HD protein at the electroporated side and the number of cells expressing the endogenous Lim-HD protein at the control side were counted. The box plots show the ratio between them. Comparing each experimental group to the control (nGFP) using Dunnett's method (which takes into account multiple comparisons) shows a significant difference between the control group and the experimental groups. The circle charts show the significance of these results. № overlapping between the control group (green circle) and the experimental groups (black circles) is indicative of $p$ values $<0.05$. Scale bar in $\boldsymbol{D}$ is $200 \mathrm{~mm}$.

projected laterally, dorsally to the motor neuron zone (Figs. 4, $8 F)$. At E6, dII $1^{\text {Isll/taumyc }}$ punctuated staining at the marginal zone, above the motor neuron, is observed (Fig. $8 G$ ). Thus, dI $1_{\text {ipsi }}$ neurons are not affected by the ectopic expression of Isl1.

Inspection of the dorsal spinal cord by open-book preparation and cross sections of $\mathrm{dI} 1^{\text {Isl1/taumyc }}$ embryos revealed that the characteristic $\mathrm{dI} 3_{\text {dorsal }}$ fascicule is not apparent. Thus, the only change in the axonal trajectories of $\mathrm{dI} 1^{\mathrm{Isl} 1 / \text { taumyc }}$ is the lateral deflection, along the $\mathrm{dI} 3_{\text {ventral }}$ axonal route, of $\mathrm{dI} 1_{\text {comm }}$ from the projection toward the floor plate.

\section{Discussion}

In the current study, we have combined molecular and morphological tools to follow the development and axonal patterning of a molecularly defined group of dorsal spinal interneurons-dI3 neurons. By exploiting newly characterized enhancer elements, combined with an enhancer-intersection method, the axonal pathways of $\mathrm{dI} 3$ neurons in the chick spinal cord were revealed. Unique projection patterns were found: (1) the dorsal projection of $\mathrm{dI} 3_{\text {dorsal }}$ axons; (2) the longitudinal turn of $\mathrm{dI} 3_{\text {dorsal }}$ axons at the DF; and (3) the lateral turn of $\mathrm{dI} 3_{\text {ventral }}$ axons at the ventral neural tube. Two new checkpoints of axonal turning were found: the axons of motor and DRG neurons. We provide evidence that the Lim-HD protein Isl1 is not required for the acquisition of $\mathrm{dI} 3$ fate, but is sufficient to impose ipsilateral turning along the motor axons to the commissural $\mathrm{dI} 1_{\text {comm }}$ neurons.

\section{Do dI3 neurons relay nociceptive sensory information?}

dI3 and dI5 neurons are considered to be nociceptive secondorder sensory neurons (Qian et al., 2002; Xu et al., 2008). During early embryonic development, both populations express transcription factors that are shared by the primary afferent and second-order nociceptive neurons, Tlx3 and PrrxL1, and both express pain-modulatory neuropeptides (Qian et al., 2002; Xu et al., 2008). However, while the adult dI5 share numerous characteristics that support their role as nociceptive relay neurons, there is less evidence to support a similar role for dI 3 neurons. Most of dI5 neurons migrate dorsally to laminas I and II, laminas that are 

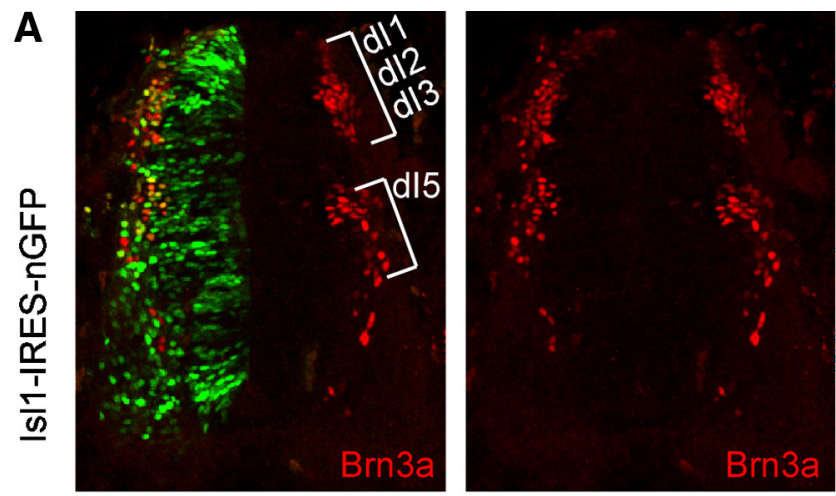

B
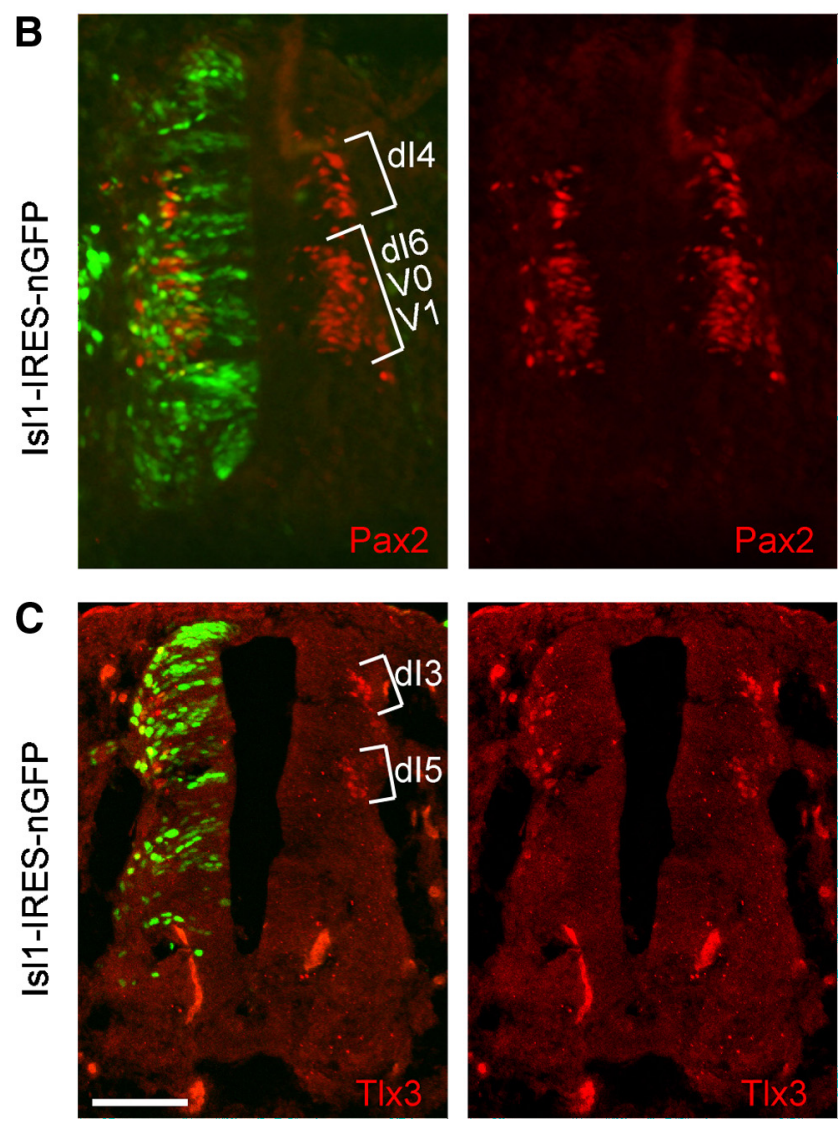

Figure 6. Ectopic expression of IsI1 does not alter neuronal cell fate. Ectopic IsI1 does not change the pattern of Brn3a $(\boldsymbol{A}), \operatorname{Pax} 2(\boldsymbol{B})$, and Tlx3 $(\boldsymbol{C})$. The images on the left show the overlapping between the electroporated cells and the cell fate marker-positive neurons. The images on the right show the cell fate marker channel. Scale bar in C is $200 \mathrm{~mm}$.

innervated by the coetaneous $\mathrm{A} \delta$ and C fibers, while $\mathrm{dI} 3$ neurons and a portion of dI5 neurons that settle in deep dorsal horn laminas migrate ventrally. The expression of Tlx3 and PrrxL1 in dI5 neurons (in the dorsal lamina) persists through postgestation, while their expression in $\mathrm{dI} 3$ and the ventral dI5 is transient-only during embryogenesis. Numerous genes that encode for neuropeptides are expressed in the dorsal dI5: Tac2, Tac1, CCK, and Sst, while dI 3 and the ventral dI5 neurons express only CCK and Tacl (Qian et al., 2002; Xu et al., 2008). The axonal trajectory pathways of $\mathrm{dI} 3$ neurons, as revealed in the current study, also undermine their possible role in relaying pain. dI3 neurons are ipsilaterally projecting neurons, while most of the nociceptive information from the spinal cord to the thalamus and the cerebral cortex is projected contralaterally within the spinal cord. dI3 may still belong to the spinoreticular, spinocervical, or polysynaptic dorsal column pathways tract, since neurons that project in this tract are ventrally located, and many of them do not cross the midline (Millan, 1999). Some of the layer 5 interneurons integrate information from $\mathrm{A} \delta$ and $\mathrm{A} \beta$ fibers. The final position of $\mathrm{dI} 3$ neurons in the adult spinal cord, as well as their afferent input, is not known. Examination of the soma position, afferent input, and the possible innervation of the reticular formation of the medulla by dI3 axons at later developmental stages may shed light on the possible role of $\mathrm{dI} 3$ neurons in the nociceptive circuit.

\section{The axons of DRG and motor neurons as en passant} guidance cues

The assembly of neuronal circuits is governed by a series of interactions between the growth cones and intermediate cues arrayed along their axonal pathway and their putative cellular target (Tessier-Lavigne and Goodman, 1996; Yu and Bargmann, 2001). In the developing spinal cord, the neuroepithelial midline group of cells, the floor plate and the roof plate, has a profound role in patterning axonal trajectory of the spinal interneurons and motor neurons. The roof plate serves as a guidepost cue that directs the initial ventral growth of the dorsal commissural interneurons by secreting a long-range repulsive signal (Augsburger et al., 1999; Butler and Dodd, 2003). The floor plate, an intermediate target, is a source of short- and long-range attractive cues that guide the commissural axons toward and across the floor plate (Tessier-Lavigne et al., 1988; Charron et al., 2003), and a source of short-range repulsive signals that prevent recrossing of the floor plate (Brose et al., 1999). After the midline crossing, the floor plate is a source of attractive and repulsive cues that govern the rostral turning of commissural axons (Lyuksyutova et al., 2003; Bourikas et al., 2005). Little is known about the positional cues that direct the growth of INs after assuming longitudinal growth.

Can the final architecture of the spinal interneuron tracks be attributed solely to these two cues? In Drosophila, the arrangement of the three longitudinal tracks along the medial/lateral axis of the CNS is mediated by the Slit repulsive signal emanating from the midline glial cells (Rajagopalan et al., 2000; Simpson et al., 2000). Slit may exert a similar long-range repulsive signal that determines the position of the longitudinal fascicules along the ventral/dorsal axis also in vertebrates. Expression of a dominant-negative form of the Slit receptors Robol and Robo 2 in $\mathrm{dI} 1$ and $\mathrm{dI} 2$ neurons prevents deflection from the floor plate of the postcrossing axons. The manipulated axons subsequently elongate along the floor plate boundary rather than at the lateral funiculus (Reeber et al., 2008).

In the current study, we demonstrate two novel positional cues, the axons of DRG and motor neurons, that may determine the position of $\mathrm{dI} 3$ axons at the VLF and DF. Initially, $\mathrm{dI} 3_{\text {dorsal }}$ neurons may either be repelled or attracted by long-range guidance signals emanating from the floor plate or the roof plate, respectively. However, when $\mathrm{dI} 3_{\text {dorsal }}$ axons reach the axons of DRG neurons at the DREZ, they turn longitudinally and join their axons at the DF. Likewise, the axons of $\mathrm{dI} 3_{\text {ventral }}$ neurons are initially oriented toward the floor plate. As they encounter the zone occupied by motor neurons, they turn laterally with the motor axons. The requirement for close contact between $\mathrm{dI} 3_{\text {ventral }}$ and motor axons is further demonstrated in the axonal trajectory of Isl1 ectopically expressing dI1 neurons. The axons of $\mathrm{dI} 1_{\text {ipsi }}$ neurons turn laterally and dorsally to the motor neurons. Thus, they are never exposed to short-range motor neuron-derived signals. Thus, ectopic expression of Isl1 in 

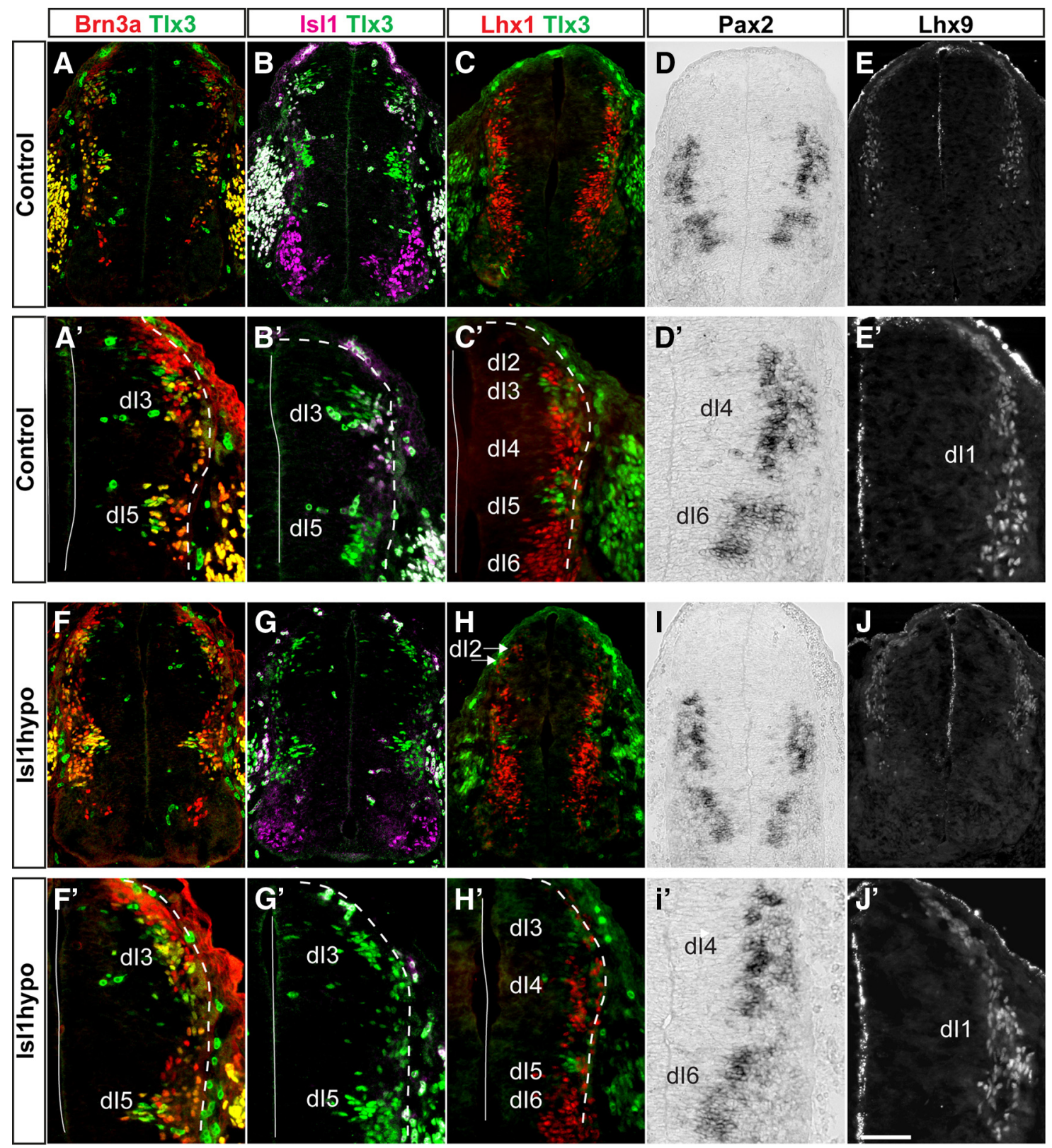

Figure 7. Isl1 is not required for dl3 cell fate. Cross sections of an E11.5 embryo of $\mid$ s| 1 hypo $(\boldsymbol{F}-\boldsymbol{J})$ and littermate 111.5 embryo $(\boldsymbol{A}-\boldsymbol{E})$ were stained with antibodies to Brn3a $(\boldsymbol{A}, \boldsymbol{F})$, T|x3 $(\boldsymbol{A}-\boldsymbol{C}, \boldsymbol{F}-\boldsymbol{H})$, $|s| 1(\boldsymbol{B}, \boldsymbol{G}), \operatorname{Lhx} 1 / 5(\boldsymbol{C}, \boldsymbol{H})$, and $\operatorname{Lhx}(\boldsymbol{E}, \boldsymbol{J})$; and with mRNA probe for $\operatorname{Pax2}(\boldsymbol{D}, \boldsymbol{I})$. $\boldsymbol{A}$-J are entire neural tube images, $\boldsymbol{A}^{\prime}-J^{\prime}$ are magnifications of the dorsal half of hemineural tubes. Sections were triple stained with Brn3a, Tlx3, and Is|1. Isl1 is not expressed in the dorsal neural tube of Is11hypo (compare $\boldsymbol{B}$ and $\boldsymbol{B}^{\prime}$ to $\mathbf{G}$ and $\boldsymbol{G}^{\prime}$ ). No change is evident in the expression pattern of Brn3a, Tlx3, Lhx1, Pax2, or Lhx9. Scale bar in $\boldsymbol{F}$ is $300 \mu \mathrm{m}$ for $\boldsymbol{A}-J$ and $150 \mu \mathrm{m}$ for $\boldsymbol{A}^{\prime}-\boldsymbol{J}^{\prime}$.

dI $1_{\text {ipsi }}$ does not alter their axonal patterning. Ectopic expression of Isl1 in $\mathrm{dI} 1_{\text {comm }}$ does not change their dorsal soma position, or their initial ventral trajectory. They are, however, deflected laterally when they encounter the motor neuron zone, thus supporting the hypothesis that short-range adhesive interaction between motor axons and dI3 axons is required for lateral turning.

The requirement of DRG and motor axons for the axonal trajectory of dI3 neurons at these choice points can be challenged by manipulating DRG axons and motor neurons.

\section{The role of Isl1 in dI 3 neurons}

A Lim code distinguishes between the three dorsal IN: dI1-3. Loss-of-function experiments have demonstrated that Lhx2/9 and Lhx $1 / 5$ are not required for cell fate acquisition of dI 1 and dI 2 neurons (Pillai et al., 2007; Wilson et al., 2008). In the current study, we demonstrate that Isll is likewise not required for the acquisition of $\mathrm{dI} 3$ cell fate. The cross-repression interactions between Lim-HD proteins in the dorsal IN are likely to serve as a maintenance mechanism to ensure cell fate, while the bHLH proteins Atoh1, Ngn1/2, and Ascl1 are the cell fate determination factors. A similar role for Isll in the early acquisition of cell fate in the peripheral nervous system has been demonstrated. The development of the trigeminal and DRG ganglia is grossly normal in the absence of Isl1; however, the expression of Lhx2 and Lhx1 is induced ectopically in DRG neurons (Sun et al., 2008), supporting a role for Isl1 in the repression of other Lim-HD genes. Our gain-offunction experiments suggest that Isll is involved in one of the later stages of dI3 differentiation-axonal patterning. A similar role for Lhx2 and Lhx9 in the differentiation of dI1 neurons was demon- 


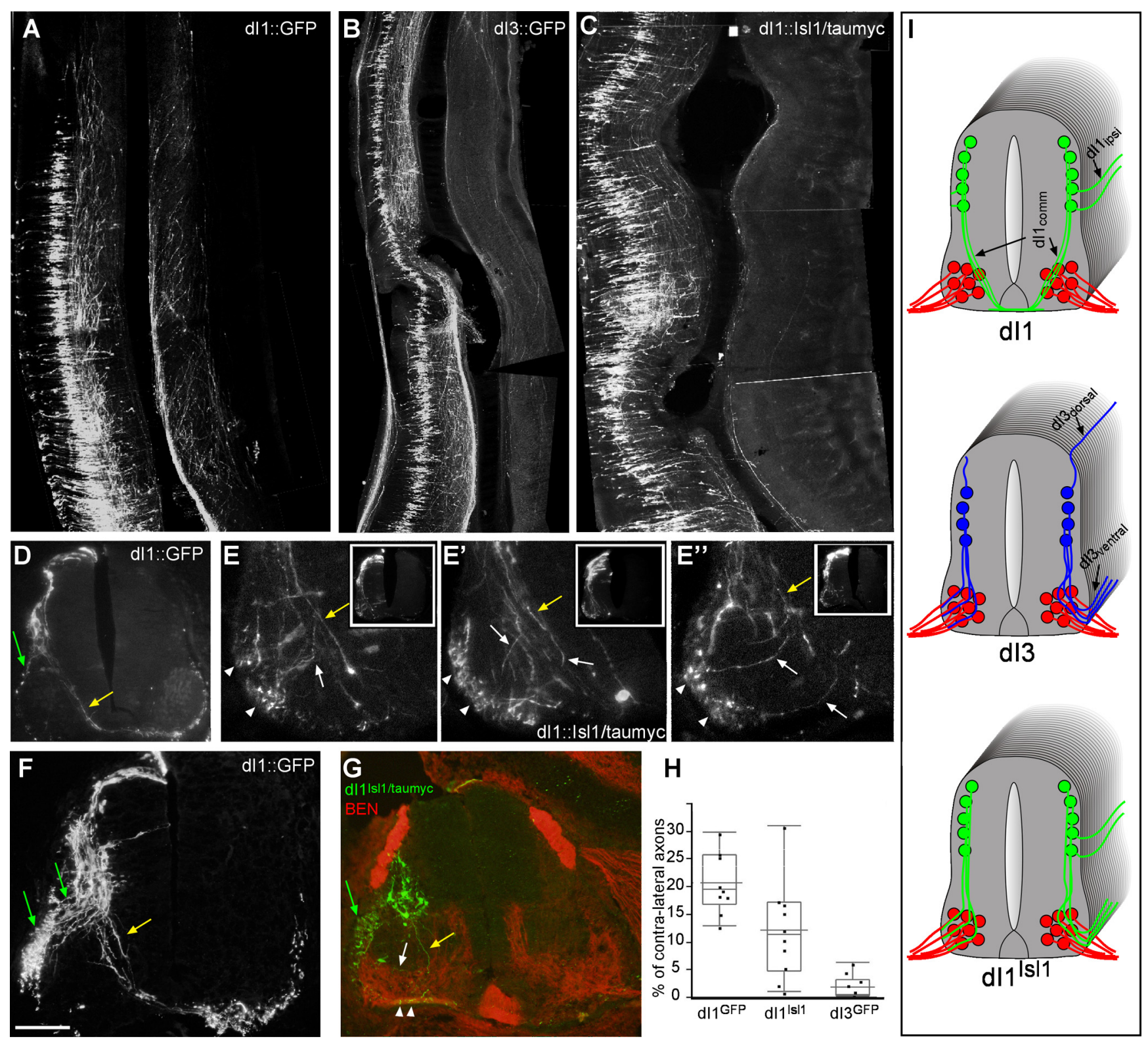

Figure 8. Ectopic Is 11 instructs a lateral turning to the commissural dl1 1 axons at the motor neuron choice point. Is $\mid 1$-IRES-taumyc $(C, E, G)$ and GFP $(A, D, F)$ were expressed in d|1 neurons using the Cre/LoxP and Edl1 enhancers. GFP was expressed in dl 3 neurons $(\boldsymbol{B})$ as described in Figure 2 . In $\boldsymbol{D}$, the yellow arrow points to $\mathrm{dl} 1_{\text {comm }}$ and the green arrow to d $11_{\text {ipsi. }}$. In $\boldsymbol{E}$, the yellow arrows point to the ventral projection, the white arrows point to the laterally turning $\mathrm{d} 11^{\text {Is/taumyc }}$ axons, and the arrowheads point to the longitudinally turning $\mathrm{d} 1{ }^{1 \text { s/ltaumyc }}$ axons. The insets in $\boldsymbol{E}$ show the entire neural tube, while the image shows the ventral lateral side of the electroporated side. At E6, the ipsilateral projection of $\mathrm{dl}_{1}{ }_{\text {ipsi }}$ is apparent $(\boldsymbol{F}$, green arrows). Ectopic expression of $\mathrm{Is} \mid 1$ in $\mathrm{d} \mid 1 \mathrm{neurons}$ does not change the projection pattern of $\mathrm{dl}_{1} 1_{\text {ipsi }}(\boldsymbol{G})$. $\mathrm{dl} 1_{\text {ipsi }}$ axons are projected laterally (green arrow in $\mathbf{G}$ ) and dorsally to the motor neuron $(\boldsymbol{G}$, stained with BEN antibody). The percent of axons crossing to the contralateral side from the total axons is shown in $\boldsymbol{H}$. The area occupied by the axons at the ipsilateral and contralateral sides of electroporated open-book preparations was measured using ImageJ software. Three open books for each group, and 3-4 different levels from each open book, were measured. The Wilcoxon/Kruskal-Wallis test revealed a significant difference between the groups ( $p<0.001$ ). A scheme that demonstrates the axonal projection patterns of dl1, dl3, and dl1 1 sl1 neurons is presented in $I$. Scale bar in $\boldsymbol{B}$ is $300 \mathrm{~mm}$ for $\boldsymbol{A}, 350 \mu \mathrm{m}$ for $\boldsymbol{B}, 250 \mu \mathrm{m}$ for $\boldsymbol{C}$, $300 \mu \mathrm{m}$ for $\boldsymbol{D}, 150 \mu \mathrm{m}$ for $\boldsymbol{E}$, and $200 \mu \mathrm{m}$ for $\boldsymbol{F}$ and $\boldsymbol{G}$.

strated. They are not required for cell differentiation, but rather, for dI1 axonal projection (Wilson et al., 2008; Avraham et al., 2009).

Motor, DRG, and dI3 neurons express Isl1. Thus, Isl1 may regulate homophilic interaction between Isl1-expressing neurons. Corroborating this hypothesis is the contralateral-toipsilateral turn of Isl1-expressing $\mathrm{dI} 1_{\text {comm }}$ axons as they reach the motor neuron zone. The presumed Isl1-mediated homophilic interaction is likely to be transient, since $\mathrm{dI} 3_{\text {ventral }}$ axons depart from motor axons as they turn longitudinally. Other guidance cues that reside in the white matter may dominate, sequester, or inhibit the Isl1-mediated homophilic interaction. The axons of
Isl1-expressing neurons serve as an intermediate target for dI3 axons. Do dI3 axons serve, reciprocally, as guidance cues for motor and DRG neurons? The initial axonal pathway of motor neurons, laterally to the midline, and DRG axons, elongation at the DF, are initiated before the arrival of $\mathrm{dI} 3$ axons to those checkpoints. Hence, it is unlikely that dI 3 axons serve as a scaffold for the initial growth of motor and DRG axons. Accordingly, conditional removal of Isll from DRG neurons results in a specific absence of cutaneous innervation in the dorsal spinal lamina, while elongation along the longitudinal axis at the DF is not altered (Sun et al., 2008). 
Subsequently to the elongation at the DF, proprioceptive Ia/II sensory afferent axons branch from the longitudinal DF fascicule, project ventrally toward the ventral spinal cord, and synapse with motor neurons. dI3 axons, projecting to both DRG and motor axons, may serve as a scaffold for the afferent axons. In support of this, at E15.5, mouse dI3 neurons are settled along the afferent axons as they extend ventrally toward motor neurons (Ding et al., 2005). Inhibition in ventral migration of $\mathrm{dI} 3$ neurons results in a failure of ventral extension of the afferent axons (Ding et al., 2005).

Our study underscores two axonal checkpoints, of the DRG and motor neurons that serve as en passant guidance cues for $\mathrm{dI} 3$ axons. Further experiments aimed at eliminating those transient targets and downregulating Isl1 expression in dI3, motor, or DRG neurons are required to establish the requirement of motor axons, DRG axons, and Isl1 for the guidance of dI3 axons.

\section{References}

Alvarez FJ, Jonas PC, Sapir T, Hartley R, Berrocal MC, Geiman EJ, Todd AJ, Goulding M (2005) Postnatal phenotype and localization of spinal cord V1 derived interneurons. J Comp Neurol 493:177-192.

Augsburger A, Schuchardt A, Hoskins S, Dodd J, Butler S (1999) BMPs as mediators of roof plate repulsion of commissural neurons. Neuron 24:127-141.

Avraham O, Hadas Y, Vald L, Zisman S, Schejter A, Visel A, Klar A (2009) Transcriptional control of axonal guidance and sorting in dorsal interneurons by the Lim-HD proteins Lhx9 and Lhx1. Neural Dev 4:21.

Avraham O, Zisman S, Hadas Y, Vald L, and Klar A (2010) Deciphering axonal pathways of genetically defined groups of neurons in the chick neural tube utilizing in ovo electroporation. J Vis Exp 1792.

Bourikas D, Pekarik V, Baeriswyl T, Grunditz A, Sadhu R, Nardó M, Stoeckli ET (2005) Sonic hedgehog guides commissural axons along the longitudinal axis of the spinal cord. Nat Neurosci 8:297-304.

Brose K, Bland KS, Wang KH, Arnott D, Henzel W, Goodman CS, TessierLavigne M, Kidd T (1999) Slit proteins bind Robo receptors and have an evolutionarily conserved role in repulsive axon guidance. Cell 96: 795-806.

Butler SJ, Dodd J (2003) A role for BMP heterodimers in roof platemediated repulsion of commissural axons. Neuron 38:389-401.

Charron F, Stein E, Jeong J, McMahon AP, Tessier-Lavigne M (2003) The morphogen sonic hedgehog is an axonal chemoattractant that collaborates with netrin-1 in midline axon guidance. Cell 113:11-23.

Ding YQ, Kim JY, Xu YS, Rao Y, Chen ZF (2005) Ventral migration of early-born neurons requires Dcc and is essential for the projections of primary afferents in the spinal cord. Development 132:2047-2056.

Helms AW, Johnson JE (2003) Specification of dorsal spinal cord interneurons. Curr Opin Neurobiol 13:42-49.

Imondi R, Kaprielian Z (2001) Commissural axon pathfinding on the contralateral side of the floor plate: a role for B-class ephrins in specifying the dorsoventral position of longitudinally projecting commissural axons. Development 128:4859-4871.

Kania A, Jessell TM (2003) Topographic motor projections in the limb imposed by LIM homeodomain protein regulation of ephrin-A:EphA interactions. Neuron 38:581-596.
Lee KJ, Jessell TM (1999) The specification of dorsal cell fates in the vertebrate central nervous system. Annu Rev Neurosci 22:261-294.

Luria V, Krawchuk D, Jessell TM, Laufer E, Kania A (2008) Specification of motor axon trajectory by ephrin-B:EphB signaling: symmetrical control of axonal patterning in the developing limb. Neuron 60:1039-1053.

Lyuksyutova AI, Lu CC, Milanesio N, King LA, Guo N, Wang Y, Nathans J, Tessier-Lavigne M, Zou Y (2003) Anterior-posterior guidance of commissural axons by Wnt-frizzled signaling. Science 302:1984-1988.

Millan MJ (1999) The induction of pain: an integrative review. Prog Neurobiol 57:1-164.

Pillai A, Mansouri A, Behringer R, Westphal H, Goulding M (2007) Lhx1 and Lhx5 maintain the inhibitory-neurotransmitter status of interneurons in the dorsal spinal cord. Development 134:357-366.

Qian Y, Shirasawa S, Chen C-L, Cheng L, Ma Q (2002) Proper development of relay somatic sensory neurons and D2/D4 interneurons requires homeobox genes Rnx/Tlx-3 and Tlx-1. Genes Dev 16:1220-1233.

Rajagopalan S, Vivancos V, Nicolas E, Dickson BJ (2000) Selecting a longitudinal pathway: Robo receptors specify the lateral position of axons in the Drosophila CNS. Cell 103:1033-1045.

Raymond CS, Soriano P (2007) High-efficiency FLP and PhiC31 sitespecific recombination in mammalian cells. PLoS One 2:e162.

Reeber SL, Sakai N, Nakada Y, Dumas J, Dobrenis K, Johnson JE, Kaprielian Z (2008) Manipulating Robo expression in vivo perturbs commissural axon pathfinding in the chick spinal cord. J Neurosci 28:8698-8708.

Simpson JH, Bland KS, Fetter RD, Goodman CS (2000) Short-range and long-range guidance by Slit and its Robo receptors: a combinatorial code of Robo receptors controls lateral position. Cell 103:1019-1032.

Song M-R, Sun Y, Bryson A, Gill GN, Evans SM, Pfaff SL (2009) Islet-toLMO stoichiometries control the function of transcription complexes that specify motor neuron and V2a interneuron identity. Development 136:2923-2932.

Sun Y, Dykes IM, Liang X, Eng SR, Evans SM, Turner EE (2008) A central role for Islet 1 in sensory neuron development linking sensory and spinal gene regulatory programs. Nat Neurosci 11:1283-1293.

Tessier-Lavigne M, Goodman CS (1996) The molecular biology of axon guidance. Science 274:1123-1133.

Tessier-Lavigne M, Placzek M, Lumsden AGS, Dodd J, Jessell TM (1988) Chemotropic guidance of developing axons in the mammalian central nervous system. Nature 336:775-778.

Visel A, Bristow J, Pennacchio LA (2007a) Enhancer identification through comparative genomics. Semin Cell Dev Biol 18:140-152.

Visel A, Minovitsky S, Dubchak I, Pennacchio LA (2007b) VISTA Enhancer Browser-a database of tissue-specific human enhancers. Nucleic Acids Res 35:D88-92.

Wilson SI, Shafer B, Lee KJ, Dodd J (2008) A molecular program for contralateral trajectory: Rig-1 control by LIM homeodomain transcription factors. Neuron 59:413-424.

Xu Y, Lopes C, Qian Y, Liu Y, Cheng L, Goulding M, Turner EE, Lima D, Ma Q (2008) Tlx1 and Tlx3 coordinate specification of dorsal horn painmodulatory peptidergic neurons. J Neurosci 28:4037-4046.

Yu TW, Bargmann CI (2001) Dynamic regulation of axon guidance. Nat Neurosci 4 [Suppl]:1169-1176.

Zisman S, Marom K, Avraham O, Rinsky-Halivni L, Gai U, Kligun G, Tzarfaty-Majar V, Suzuki T, Klar A (2007) Proteolysis and membrane capture of F-spondin generates combinatorial guidance cues from a single molecule. J Cell Biol 178:1237-1249. 